\title{
Detections of Neutron Stars in the Extreme Ultraviolet
}

\author{
Stuart Bowyer \\ Center for EUV Astrophysics, University of California, Berkeley, CA 94720-5030, USA
}

Neutron stars were not expected to be observable in the extreme ultraviolet and hence their detection in this band is a surprise. More important is that EUV observations provide unique new information on these objects. In the accompanying Table 1, I list the neutron stars detected in the EUV with the Extreme Ultraviolet Explorer ( $E U V E)$. The binary object Her $\mathrm{X}-1$ is the only object detected in the all sky survey. This is not surprising since it is the only neutron star system with sufficient intensity to be detectable in the 500 to 2000 second sky intergrations which are typical for the $E U V E$ all sky survey. All the neutron stars were detected in the $100 \AA$ (Lexan) bandpass. A more complete description of $E U V E$ and the all sky survey are provided in Bowyer and Malina (1991), and in Bowyer et al. (1994).

Table 1. Neutron Stars Detected with EUVE

\begin{tabular}{|c|c|c|c|c|c|c|}
\hline Object & $\begin{array}{c}\text { Deep } \\
\text { Survey } \\
\text { cts/sec }\end{array}$ & $\begin{array}{l}\text { Lexan } \\
\text { Survey } \\
\text { cts/sec }\end{array}$ & $\begin{array}{c}\text { Age } \\
\text { years }\end{array}$ & $\begin{array}{c}\mathrm{DM} \\
\mathrm{cm}^{-3} \mathrm{pc}\end{array}$ & $\begin{array}{c}d \\
\text { pc }\end{array}$ & References \\
\hline PSR J0437-4715 & 0.038 & & $5 \times 10^{9}$ & 2.6 & 140 & (Edelstein et al. 1995) \\
\hline PSR B0656+14 & 0.024 & & $1 \times 10^{5}$ & 14.0 & 760 & (Finley et al. 1994) \\
\hline Geminga & 0.020 & & $5 \times 10^{5}$ & & $<500$ & (Halpern 1994) \\
\hline Her X-1 & & 0.18 & $<10^{7}$ & & $<5000$ & (Vrtilek et al. 1994) \\
\hline
\end{tabular}

A phased analysis of the flux from Her X-1 (Vrtilek et al. 1994) shows that the EUV flux emanates from the acretion disc and not from the neutron star itself. While these results help elucidate the character of the disc, they are not the topic of this conference and will not be discussed further.

Timing information from $E U V E$ is routinely available to $8 \mathrm{~ms}$ accuracy, and with an easily implemented alternate mode is available to better than $1 \mathrm{~ms}$. This allows the discrimination of pulse and whole-body emission from virtually all pulsars.

The pulsar $\mathrm{B} 0656+14$ was observed for over 100000 seconds and this data has 
recently been released for general dissemination via the EUVE Public Archive. A preliminary examination of this data shows the data is not pulsed and hence the EUV flux must be thermal emission from the neutron star surface.

The X-ray and $\gamma$-ray (but not radio!) pulsar, Geminga, has been observed for 300000 seconds. This data has just been released to the scientific community via the EUVE Public Archive, but as yet no analysis of this data have been published.

The millisecond pulsar J0437-4715 was observed with EUVE in January 1994 for about 70000 seconds. An unrelated EUV source about 3.8 arc minutes away was well resolved with the $\approx 1^{\prime}$ point spread function of the telescope. Because the data were not taken in the high time resolution mode, a pulse vs. whole-body deconvolution is not possible for this data. Nonetheless, Edelstein et al. (1995) have shown that a substantial amount of information can be derived from this observation.

The observed dispersion measure of $2.65 \mathrm{pc} \mathrm{cm}^{-3}$ implies a distance of 140 pc for this pulsar. This value is consistent with optical data on the white dwarf companion star. An estimate of the hydrogen column density to this system can be obtained from the compilation of Fruscione et al. (1994); a reasonable value is $\mathrm{N}_{\mathrm{HI}}=10^{19} \mathrm{~cm}^{2}$. Combining the response of the $100 \AA$ bandpass of the deep survey telescope with absorption due to an interstellar medium associated with this hydrogen column (Rumph et al. 1994) yields an effective area weighted wavelength of $116 \AA$. This is a substantially lower energy than the X-ray data obtained for this object, and leads to new insights regarding this source.

Becker \& Trümper (1993) modeled the X-ray emission of J0437-4715 with a power-law model with $\alpha=-2.60$, and with a composite model. The composite model consisted of a black-body "hot-spot" $\left(T \sim 1.7 \times 10^{6} \mathrm{~K}\right.$, Area $=0.05$ $\mathrm{km}^{2}$ ), responsible for a possible feature at $0.8 \mathrm{keV}$, combined with a power-law source with $\alpha=-2.85$ which dominates at lower energies. The predicted EUV flux for both power laws was consistent with the observed EUV luminosity only if the absorbing column was in the narrow range of $\mathrm{N}_{H I}=(2.5 \pm 0.2) \times 10^{20}$ $\mathrm{cm}^{-2}$. This column exceeds the hydrogen column out of the Galaxy (Dickey \& Lockman 1990), and hence both of these models can be ruled out as the source of the EUV flux.

The EUV data were modeled as a black body to determine if this model could produce all the ROSAT observed X-ray flux. This could be accomplished only if the hot spot emitting area was 3 times (for $N_{\mathrm{HI}}=10^{19} \mathrm{~cm}^{-2}$ ) to 25 times (for $N_{\mathrm{HI}}=10^{20} \mathrm{~cm}^{-2}$ ) larger than the area required by the X-ray data. Edelstein et al. concluded that the observed EUV flux was not consistent with a blackbody hot-spot capable of producing the soft X-ray feature.

Edelstein et al. examined whether black-body emission could provide an alternative explanation to the 0.1 to $0.4 \mathrm{keV}$ ROSAT flux which had been attributed to power-law emission. They concluded that both the EUV flux and the X-ray flux could be explained by a black-body with a temperature $\sim 5.7 \times 10^{5} \mathrm{~K}$, an emitting area of $\sim 3 \mathrm{~km}^{2}$, and ISM absorption associated with a hydrogen column of $N_{\mathrm{HI}}=5 \times 10^{19} \mathrm{~cm}^{-2}$. 
Alternatively, the EUV flux could be independent of the X-ray flux and originate from the entire surface of the pulsar. In this case, a minimum surface temperature of $T_{\min }=1.6 \times 10^{5} \mathrm{~K}$ was found for a distance of $140 \mathrm{pc}$ and a stellar radius of $10 \mathrm{~km}$. An upper limit to the pulsar's temperature $T_{\max }=4.0 \times 10^{5} \mathrm{~K}$ was established by using the known hydrogen column out of the Galaxy (Dickey \& Lockman 1990).

Thermal EUV emission from the surface of $a \sim 5 \times 10^{9}$ year old neutron star is inconsistent with standard cooling models for neutron stars (Shibazaki \& Lamb 1989) and would require some form of reheating. Four sources for reheating were considered: (1) frictional heating at the crust-core interface through the unpinning of internal vortex lines; (2) accretion from the ISM; (3) accretion from the white dwarf companion onto the neutron star; and (4) nucleon decay catalyzed by heavy magnetic monopoles.

Theoretical work (Shibazaki \& Lamb 1989, Umeda et al. 1993) predicts that for neutron stars older than $10^{9} \mathrm{yr}$, the thermal surface temperatures from frictional heating will be less than $10^{5} \mathrm{~K}$. Hence standard frictional heating models can be ruled out.

The gravitational accretion of ISM could heat the neutron star if the velocity of the star is less than $\sim 10 \mathrm{~km} \mathrm{~s}^{-1}$ (Paczyński 1990, Hartmann et al. 1990, Treves \& Colpi 1991). A velocity of $91 \pm 3 \mathrm{~km} \mathrm{~s}^{-1}$ has been measured for PSR $0437-4715$ using timing measurements (Bell et al. 1995), and $63 \pm 30 \mathrm{~km} \mathrm{~s}^{-1}$ using scintillation observations (Johnston \& Nicastro 1995). These velocity estimates are sufficiently large that significant heating by accretion from the ISM can be ruled out.

Stellar material abated from the neutron stars' white dwarf companion by a high energy particle wind from the pulsar could accrete on the neution star and produce heating. An upper limit to mass accretion by a high-energy particle wind from the pulsar is

$$
\dot{M} \leq f L_{\mathrm{SD}} / \gamma c^{2}
$$

where $f$ is the fraction of the total flux from the neutron star intercepted by the companion, $L_{\mathrm{SD}}$ is the pulsar spin-down luminosity, $\gamma$ is the Lorentz factor of the particles, and $c$ is the speed of light. For parameters appropriate to this system the maximum luminosity due to this accretion is an insignificant fraction of the observed EUV luminosity.

It has been suggested that neutron stars could be heated by magnetic "monopole-catalyzed" nucleon decay (Freese et al. 1983, Kolb \& Turner 1984). Magnetic monopoles which hit the surface of a neutron star will be captured by the star at a rate proportional to the monopole flux. The combination of the upper limit to the surface temperature of PSR $0437-4715$ and its extreme age results in a value for the Galactic monopole flux that is three orders of magnitude lower than existing limits.

Four neutron stars (or binaries with a neutron star as one component) have been detected in the EUV to date. In three of these systems the observed emission is believed to be from the neutron star itself, but a detailed analysis has been carried out for only one of these objects. In this case substantial new information 
on neutron star physics has been obtained. We can expect more progress in this field when analysis of the existing data are completed, and as new neutron stars are found to be emitting in the EUV.

Acknowledgements: This work is being supported by NASA Grant NAS5-30180.

\section{References}

Becker W., Trümper J., 1993, Nature 365, 528

Bell J.F., Bailes M., Manchester R.N., Weisberg J.M., Lyne A.G., 1995 (submitted)

Bowyer S., Malina R.F., 1991, in Extreme Ultraviolet Astronomy, R.F. Malina and S.

Bowyer (eds.). Pergamon Press, New York, p. 397

Bowyer S., Lieu R., Lampton M., Lewis J., Wu X., Drake J.J., Malina R.F, 1994, The First EUVE Source Catalog, ApJS 93, 569

Dickey J.M., Lockman F.J., 1990, ARA\&A 28, 215

Edelstein J., Foster R.S., Bowyer S., 1995, ApJ (submitted)

Finley J.P., Ogelman H., Edelstein J., 1994, 184th AAS Bulletin

Freese K., Turner M., Schramm D.N., 1983, Phys. Rev. Lett. 51, 1625

Fruscione A., Hawkins I., Jelinsky P., Wiercigroch A., 1994, ApJS 94, 127

Halpern J., 1994, private communication

Hartmann D., Epstein R.I., Woosley S.E., 1990, ApJ 348, 625

Johnston S., Nicastro L., 1995, preprint

Kolb E., Turner M., 1984, ApJ 286, 702

Paczyński B., 1990, ApJ 348, 485

Rumph T., Bowyer S., Vennes S., 1994, AJ 107, 2108

Treves A., Colpi M., 1991, A\&A 241, 107

Shibazaki N., Lamb F.K., 1989, ApJ 346, 808

Umeda H., Shibazaki N., Nomoto K., Tsuruta S., 1993, ApJ 408, 186

Vrtilek S.D., Mihara T., Primini F.A., Kahabka P., Marshal H., Agerer F., Charles P.A., Cheng F.H., Dennerl K., la Dous C., Hu E.M., Rutten R., Serlemitsos P., Soong Y., Stull J., Trümper J., Voges W., Wagner R.M., Wilson R., 1994, ApJ 436, L9 